\title{
New transformation of Wigner operator in phase space quantum mechanics for the two-mode entangled case
}

\author{
Hong-yi Fan $^{1,2}$ and Hong-chun Yuan ${ }^{1}$ \\ ${ }^{1}$ Department of Physics, Shanghai Jiao Tong University, Shanghai, 200030, China \\ Corresponding author: yuanhch@sjtu.edu.cn \\ ${ }^{2}$ Department of Material Science and Engineering, \\ University of Science and Technology of China, Hefei, Anhui 230026, China
}

\begin{abstract}
As a natural extension of Fan's paper (arXiv: 0903.1769vl [quant-ph]) by employing the formula of operators' Weyl ordering expansion and the bipartite entangled state representation we find new two-fold complex integration transformation about the Wigner operator $\Delta(\mu, \nu)$ (in its entangled form) in phase space quantum mechanics,

$$
\begin{aligned}
& \int \frac{d^{2} \mu d^{2} \nu}{\pi^{2}} e^{(\xi-\mu)\left(\eta^{*}-\nu^{*}\right)-(\eta-\nu)\left(\xi^{*}-\mu^{*}\right)} \Delta(\mu, \nu) \\
& =\delta\left(\eta-a_{1}+a_{2}^{\dagger}\right) \delta\left(\eta^{*}-a_{1}^{\dagger}+a_{2}\right) \delta\left(\xi-a_{1}-a_{2}^{\dagger}\right) \delta\left(\xi^{*}-a_{1}^{\dagger}-a_{2}\right),
\end{aligned}
$$

and its inverse transformation, where $a_{i}, a_{i}^{\dagger}$ are bosonic creation and annihilation operators, respectively. In this way, some operator ordering problems regarding to $\left(a_{1}^{\dagger}-a_{2}\right)$ and $\left(a_{1}+a_{2}^{\dagger}\right)$ can be solved and the contents of phase space quantum mechanics can be enriched.

PACS: 03.65.-w, 02.90.+p

Keywords: Wigner operator in entangled form; Weyl ordering; two-fold complex integration transformation
\end{abstract}

\section{INTRODUCTION}

Integration transformations are very useful in mathematical physics. In the preceding paper [1] we have reported a new integration transformation in $q-p$ phase space

$$
\iint_{-\infty}^{\infty} \frac{d p d q}{\pi} e^{2 i(p-x)(q-y)} h(p, q)=f(x, y),
$$

which is invertible

$$
\iint_{-\infty}^{\infty} \frac{d x d y}{\pi} e^{-2 i(p-x)(q-y)} f(x, y)=h(p, q)
$$

and proved that this transformation obeys Parseval theorem. By virtue of the formula of operators' Weyl ordering expansion [2, 3], we find the following new two-fold $q$-number integration transformation about the Wigner operator $\Delta\left(q^{\prime}, p^{\prime}\right)[4,5,6]$ in phase space quantum mechanics [7],

$$
\iint_{-\infty}^{\infty} \frac{\mathrm{d} p^{\prime} \mathrm{d} q^{\prime}}{\pi} \Delta\left(q^{\prime}, p^{\prime}\right) e^{-2 i\left(p-p^{\prime}\right)\left(q-q^{\prime}\right)}=\delta(p-P) \delta(q-Q),
$$

and its inverse

$$
\iint_{-\infty}^{\infty} \mathrm{d} q \mathrm{~d} p \delta(p-P) \delta(q-Q) e^{2 i\left(p-p^{\prime}\right)\left(q-q^{\prime}\right)}=\Delta\left(q^{\prime}, p^{\prime}\right),
$$

where $Q, P$ are the coordinate and momentum operators, respectively. $[Q, P]=i \hbar$. These two equations can be applied to studying mutual converting formulas among $Q-P$ ordering, $P-Q$ ordering and Weyl ordering of operators.

In the present paper, we extend Eqs.(3) and (44) to the two-mode entangled case and construct the two-fold complex integration transformation about the Wigner operator $\Delta(\mu, \nu)$ (in its entangled form, $\mu, \nu$ are complex) in phase space quantum mechanics. By mentioning two-mode entangled case, we naturally think of two mutually conjugate entangled state representations $|\xi\rangle$ and $|\eta\rangle$ (see Sect. 2 below). In Sect. 3, after deriving the Weyl ordering of $|\eta\rangle\langle\xi|$ by virtue of the formula of operator's Weyl ordering expansion, we give the Weyl ordering form of 
$\delta\left(\nu-a_{1}+a_{2}^{\dagger}\right) \delta\left(\nu^{*}-a_{1}^{\dagger}+a_{2}\right) \delta\left(\mu-a_{1}-a_{2}^{\dagger}\right) \delta\left(\mu^{*}-a_{1}^{\dagger}-a_{2}\right)$, where $a_{i}, a_{i}^{\dagger}$ are the bosonic creation and annihilation operators satisfying $\left[a_{i}, a_{j}^{\dagger}\right]=\delta_{i j}$. Then in Sect. 4 , we find a new two-fold complex integration transformation relating the Wigner operator $\Delta(\mu, \nu)$ and the operator $\delta\left(\nu-a_{1}+a_{2}^{\dagger}\right) \delta\left(\nu^{*}-a_{1}^{\dagger}+a_{2}\right) \delta\left(\mu-a_{1}-a_{2}^{\dagger}\right)$ $\delta\left(\mu^{*}-a_{1}^{\dagger}-a_{2}\right)$. In Sect. 5 we tackle some operator ordering problems regarding to $\left(a_{1}^{\dagger}-a_{2}\right)$ and $\left(a_{1}+a_{2}^{\dagger}\right)$. In this way, the contents of phase space quantum mechanics can be further enriched.

\section{BRIEF REVIEW OF THE TWO MUTUALLY CONJUGATE ENTANGLED STATES}

The conception of quantum entanglement has been paid much attention because of their wide uses in quantum communication and quantum computation. The original concept of quantum entanglement pointed out by Einstein, Podolsky and Rosen (EPR) in their famous paper [8] arguing the incompleteness of quantum mechanics is for a bipartite system characteristic of continuous variables. According to the original idea of EPR that two particles' relative coordinate operator commutes with their total momentum operator, $\left[Q_{1}-Q_{2}, P_{1}+P_{2}\right]=0$, we have set up the bipartite entangle state representation of continuous variable in two-mode Fock space [9], which is the common eigenvector $|\eta\rangle$ of $Q_{1}-Q_{2}$ and $P_{1}+P_{2}$, i.e.

$$
|\eta\rangle=\exp \left(-\frac{1}{2}|\eta|^{2}+\eta a_{1}^{\dagger}-\eta^{*} a_{2}^{\dagger}+a_{1}^{\dagger} a_{2}^{\dagger}\right)|00\rangle
$$

where $\eta \equiv \eta_{1}+i \eta_{2}$ is a complex number, $|00\rangle$ is the two-mode vacuum state. Using $\left[a_{i}, a_{j}^{\dagger}\right]=\delta_{i j}$, it can be shown that $|\eta\rangle$ obeys the eigenvector equations

$$
\left(a_{1}-a_{2}^{\dagger}\right)|\eta\rangle=\eta|\eta\rangle, \quad\left(a_{2}-a_{1}^{\dagger}\right)|\eta\rangle=-\eta^{*}|\eta\rangle
$$

$\eta^{\prime} s$ real and imaginary part are respectively the eigenvalue of $Q_{1}-Q_{2}$ and $P_{1}+P_{2}$,

$$
\left(Q_{1}-Q_{2}\right)|\eta\rangle=\sqrt{2} \eta_{1}|\eta\rangle,\left(P_{1}+P_{2}\right)|\eta\rangle=\sqrt{2} \eta_{2}|\eta\rangle
$$

$|\eta\rangle$ is proved to be complete relation and orthonormal property

$$
\int \frac{d^{2} \eta}{\pi}|\eta\rangle\langle\eta|=1
$$

and

$$
\left\langle\eta^{\prime} \mid \eta\right\rangle=\pi \delta\left(\eta^{\prime}-\eta\right) \delta\left(\eta^{\prime *}-\eta^{*}\right) \equiv \pi \delta^{(2)}\left(\eta^{\prime}-\eta\right)
$$

On the other hand, $|\eta\rangle^{\prime}$ s conjugate state, which is the common eigenvector of two particles' center-of-mass coordinate and relative momentum, is

$$
|\xi\rangle=\exp \left(-\frac{|\xi|^{2}}{2}+\xi a_{1}^{\dagger}+\xi^{*} a_{2}^{\dagger}-a_{1}^{\dagger} a_{2}^{\dagger}\right)|00\rangle,
$$

with $\xi=\xi_{1}+i \xi_{2} \cdot|\xi\rangle$ obeys

$$
\left(a_{1}+a_{2}^{\dagger}\right)|\xi\rangle=\xi|\xi\rangle, \quad\left(a_{1}^{\dagger}+a_{2}\right)|\xi\rangle=\xi^{*}|\xi\rangle
$$

and

$$
\left(Q_{1}+Q_{2}\right)|\xi\rangle=\sqrt{2} \xi_{1}|\xi\rangle,\left(P_{1}-P_{2}\right)|\xi\rangle=\sqrt{2} \xi_{2}|\xi\rangle
$$

$|\xi\rangle$ is completeness and orthonormal too,

$$
\int \frac{d^{2} \xi}{\pi}|\xi\rangle\langle\xi|=1
$$


and

$$
\left\langle\xi \mid \xi^{\prime}\right\rangle=\pi \delta\left(\xi-\xi^{\prime}\right) \delta\left(\xi^{*}-\xi^{*}\right)
$$

It is found that $|\xi\rangle$ and $|\eta\rangle$ are of equal importance because

$$
\langle\eta \mid \xi\rangle=\frac{1}{2} \exp \left[\left(\eta^{*} \xi-\xi^{*} \eta\right) / 2\right]
$$

which is just a complex form of the Fourier transformation, since $\left(\eta^{*} \xi-\xi^{*} \eta\right)$ is pure imaginary, $\langle\eta \mid \xi\rangle$ is a Fourier transform kernel.

\section{THE WEYL ORDERING FORM OF $\delta\left(\nu-a_{1}+a_{2}^{\dagger}\right) \delta\left(\nu^{*}-a_{1}^{\dagger}+a_{2}\right) \delta\left(\mu-a_{1}-a_{2}^{\dagger}\right) \delta\left(\mu^{*}-a_{1}^{\dagger}-a_{2}\right)$}

Using the complete relation Eqs.(8) and (13), as well as Eqs.(6), (11) and (15), we have

$$
\begin{aligned}
& \delta\left(\nu-a_{1}+a_{2}^{\dagger}\right) \delta\left(\nu^{*}-a_{1}^{\dagger}+a_{2}\right) \delta\left(\mu-a_{1}-a_{2}^{\dagger}\right) \delta\left(\mu^{*}-a_{1}^{\dagger}-a_{2}\right) \\
& =\int \frac{d^{2} \eta d^{2} \xi}{\pi^{2}} \delta^{(2)}\left(\nu-a_{1}+a_{2}^{\dagger}\right)|\eta\rangle\langle\eta \mid \xi\rangle\langle\xi| \delta^{(2)}\left(\mu-a_{1}-a_{2}^{\dagger}\right) \\
& =\frac{1}{2} \int \frac{d^{2} \eta d^{2} \xi}{\pi^{2}}|\eta\rangle\langle\xi| e^{\left(\eta^{*} \xi-\eta \xi^{*}\right) / 2} \delta^{(2)}(\nu-\eta) \delta^{(2)}(\mu-\xi) \\
& =\frac{1}{2}|\eta\rangle_{\eta=\nu}\left\langle\left.\xi\right|_{\xi=\mu} e^{\left(\nu^{*} \mu-\nu \mu^{*}\right) / 2} .\right.
\end{aligned}
$$

where we have simplified the notation of the product of two Delta functions

$$
\begin{aligned}
& \delta^{(2)}\left(\nu-a_{1}+a_{2}^{\dagger}\right) \delta^{(2)}\left(\mu-a_{1}-a_{2}^{\dagger}\right) \\
& \equiv \delta\left(\nu-a_{1}+a_{2}^{\dagger}\right) \delta\left(\nu^{*}-a_{1}^{\dagger}+a_{2}\right) \delta\left(\mu-a_{1}-a_{2}^{\dagger}\right) \delta\left(\mu^{*}-a_{1}^{\dagger}-a_{2}\right) .
\end{aligned}
$$

From Eq.(16) we see that once the Weyl ordering of $|\eta\rangle\langle\xi|$ is known, the Weyl ordering of $\delta\left(\nu-a_{1}+a_{2}^{\dagger}\right)$ $\delta\left(\nu^{*}-a_{1}^{\dagger}+a_{2}\right) \delta\left(\mu-a_{1}-a_{2}^{\dagger}\right) \delta\left(\mu^{*}-a_{1}^{\dagger}-a_{2}\right)$ is obtained. For this purpose, we recall the Weyl ordered expansion formula of two-mode operators [2, 3]

$$
\rho=4 \int \frac{d^{2} \beta_{1} d^{2} \beta_{2}}{\pi^{2}}:\left\langle-\beta_{1},-\beta_{2}|\rho| \beta_{1}, \beta_{2}\right\rangle \exp \left[2 \sum_{k=1}^{2}\left(\beta_{k}^{*} a_{k}-a_{k}^{\dagger} \beta_{k}+a_{k}^{\dagger} a_{k}\right)\right]:
$$

where :: denotes the Weyl ordering, within which $a_{i}, a_{i}^{\dagger}$ can be permuted, $\left|\beta_{1}, \beta_{2}\right\rangle \equiv\left|\beta_{1}\right\rangle\left|\beta_{2}\right\rangle$ is a two-mode coherent state, $\left|\beta_{i}\right\rangle=\exp \left[-\frac{1}{2}\left|\beta_{i}\right|^{2}+\beta_{i} a_{i}^{\dagger}\right]\left|0_{i}\right\rangle[10]$. For the operator $|\eta\rangle\langle\xi|$, using Eq.(18) and the overlaps

$$
\left\langle\xi \mid \beta_{1}, \beta_{2}\right\rangle=\exp \left(-\frac{1}{2}|\xi|^{2}+\xi^{*} \beta_{1}+\xi \beta_{2}-\beta_{1} \beta_{2}-\frac{1}{2}\left|\beta_{1}\right|^{2}-\frac{1}{2}\left|\beta_{2}\right|^{2}\right),
$$

and

$$
\left\langle-\beta_{1},-\beta_{2} \mid \eta\right\rangle=\exp \left(-\frac{1}{2}|\eta|^{2}-\eta \beta_{1}^{*}+\eta^{*} \beta_{2}^{*}+\beta_{1}^{*} \beta_{2}^{*}-\frac{1}{2}\left|\beta_{1}\right|^{2}-\frac{1}{2}\left|\beta_{2}\right|^{2}\right),
$$

we can derive the Weyl ordering of $|\eta\rangle\langle\xi|$ in the way 


$$
\begin{aligned}
|\eta\rangle\langle\xi| & =4 \int \frac{d^{2} \beta_{1} d^{2} \beta_{2}}{\pi^{2}}:\left\langle-\beta_{1},-\beta_{2} \mid \eta\right\rangle\left\langle\xi \mid \beta_{1}, \beta_{2}\right\rangle \exp \left[2 \sum_{k=1}^{2}\left(\beta_{k}^{*} a_{k}-a_{k}^{\dagger} \beta_{k}+a_{k}^{\dagger} a_{k}\right)\right]: \\
& =4 \int \frac{d^{2} \beta_{1} d^{2} \beta_{2}}{\pi^{2}}: \exp \left[-\frac{|\eta|^{2}}{2}-\eta \beta_{1}^{*}+\eta^{*} \beta_{2}^{*}+\beta_{1}^{*} \beta_{2}^{*}-\left|\beta_{1}\right|^{2}-\left|\beta_{2}\right|^{2}\right. \\
& \left.-\frac{|\xi|^{2}}{2}+\xi^{*} \beta_{1}+\xi \beta_{2}-\beta_{1} \beta_{2}+2 \sum_{k=1}^{2}\left(\beta_{k}^{*} a_{k}-a_{k}^{\dagger} \beta_{k}+a_{k}^{\dagger} a_{k}\right)\right]: \\
& =4 e^{-\frac{|\eta|^{2}}{2}-\frac{|\xi|^{2}}{2} \int \frac{d^{2} \beta_{2}}{\pi}:}: \exp \left[-2\left|\beta_{2}\right|^{2}+\beta_{2}\left(\xi-2 a_{2}^{\dagger}-2 a_{1}+\eta\right)\right. \\
& \left.+\beta_{2}^{*}\left(\eta^{*}+2 a_{2}+\xi^{*}-2 a_{1}^{\dagger}\right)+\left(\xi^{*}-2 a_{1}^{\dagger}\right)\left(2 a_{1}-\eta\right)+2 \sum_{k=1}^{2} a_{k}^{\dagger} a_{k}\right]: \\
& =2: \exp \left[\frac{1}{2}\left(\xi \eta^{*}-\eta \xi^{*}\right)+\xi\left(a_{2}-a_{1}^{\dagger}\right)+\eta\left(a_{2}+a_{1}^{\dagger}\right)\right. \\
& \left.-\eta^{*}\left(a_{1}+a_{2}^{\dagger}\right)+\xi^{*}\left(a_{1}-a_{2}^{\dagger}\right)+2 a_{2}^{\dagger} a_{1}^{\dagger}-2 a_{2} a_{1}\right]:
\end{aligned}
$$

where we have used the integral formula

$$
\int \frac{d^{2} z}{\pi} \exp \left(\zeta|z|^{2}+\xi z+\eta z^{*}\right)=-\frac{1}{\zeta} \exp \left[-\frac{\xi \eta}{\zeta}\right], \operatorname{Re}(\xi)<0
$$

It then follows from Eq.(21) that

$$
\frac{1}{2}|\eta\rangle\langle\xi| e^{\left(\eta^{*} \xi-\eta \xi^{*}\right) / 2}=: \exp \left[\left(\xi-a_{1}-a_{2}^{\dagger}\right)\left(\eta^{*}-a_{1}^{\dagger}+a_{2}\right)-\left(\eta-a_{1}+a_{2}^{\dagger}\right)\left(\xi^{*}-a_{1}^{\dagger}-a_{2}\right)\right]_{:}^{:}:
$$

Substituting Eq.(23) into Eq.(16) we have the Weyl ordered form

$$
\begin{aligned}
& \delta\left(\nu-a_{1}+a_{2}^{\dagger}\right) \delta\left(\nu^{*}-a_{1}^{\dagger}+a_{2}\right) \delta\left(\mu-a_{1}-a_{2}^{\dagger}\right) \delta\left(\mu^{*}-a_{1}^{\dagger}-a_{2}\right) \\
& =: \exp \left[\left(\mu-a_{1}-a_{2}^{\dagger}\right)\left(\nu^{*}-a_{1}^{\dagger}+a_{2}\right)-\left(\nu-a_{1}+a_{2}^{\dagger}\right)\left(\mu^{*}-a_{1}^{\dagger}-a_{2}\right)\right]^{:},
\end{aligned}
$$

Noting

$$
\left[a_{1}^{\dagger}-a_{2}, a_{1}+a_{2}^{\dagger}\right]=-2,
$$

we should consider another ordering other than Eq.(24), i.e.,

$$
\begin{aligned}
& \delta\left(\mu-a_{1}-a_{2}^{\dagger}\right) \delta\left(\mu^{*}-a_{1}^{\dagger}-a_{2}\right) \delta\left(\nu-a_{1}+a_{2}^{\dagger}\right) \delta\left(\nu^{*}-a_{1}^{\dagger}+a_{2}\right) \\
& =\frac{1}{2} \int \frac{d^{2} \eta d^{2} \xi}{\pi^{2}}|\xi\rangle\langle\eta| e^{\left(\eta \xi^{*}-\eta^{*} \xi\right) / 2} \delta^{(2)}(\nu-\eta) \delta^{(2)}(\mu-\xi) .
\end{aligned}
$$

Similar in the way of deriving Eq.(23), we deduce

$$
\begin{gathered}
\frac{1}{2}|\xi\rangle\langle\eta| e^{\left(\eta \xi^{*}-\eta^{*} \xi\right) / 2}=: \exp \left[\left(\eta-a_{1}+a_{2}^{\dagger}\right)\left(\xi^{*}-a_{1}^{\dagger}-a_{2}\right)-\left(\xi-a_{1}-a_{2}^{\dagger}\right)\left(\eta^{*}-a_{1}^{\dagger}+a_{2}\right)\right]: \\
: \\
: \exp \left[\left(\eta-a_{1}+a_{2}^{\dagger}\right)\left(\xi^{*}-a_{1}^{\dagger}-a_{2}\right)-\left(\eta^{*}-a_{1}^{\dagger}+a_{2}\right)\left(\xi-a_{2}^{\dagger}-a_{1}\right)\right]: \\
:
\end{gathered}
$$

It then follows

$$
\begin{aligned}
& \delta\left(\mu-a_{1}-a_{2}^{\dagger}\right) \delta\left(\mu^{*}-a_{1}^{\dagger}-a_{2}\right) \delta\left(\nu-a_{1}+a_{2}^{\dagger}\right) \delta\left(\nu^{*}-a_{1}^{\dagger}+a_{2}\right) \\
& \quad: \quad: \exp \left[\left(\nu-a_{1}+a_{2}^{\dagger}\right)\left(\mu^{*}-a_{1}^{\dagger}-a_{2}\right)-\left(\mu-a_{1}-a_{2}^{\dagger}\right)\left(\nu^{*}-a_{1}^{\dagger}+a_{2}\right)\right]:
\end{aligned}
$$




\section{THE MUTUAL INTEGRATION TRANSFORMATION BETWEEN $\delta\left(\nu-a_{1}+a_{2}^{\dagger}\right) \delta\left(\nu^{*}-a_{1}^{\dagger}+a_{2}\right)$ $\delta\left(\mu-a_{1}-a_{2}^{\dagger}\right) \delta\left(\mu^{*}-a_{1}^{\dagger}-a_{2}\right)$ AND THE WIGNER OPERATOR}

In Ref. [11] for correlated two-body systems, we have successfully derived the Wigner operator in entangled form, expressed in the entangled state $\langle\eta|$ representation as

$$
\Delta(\mu, \nu)=\int \frac{d^{2} \eta}{\pi^{3}}|\nu-\eta\rangle\langle\nu+\eta| e^{\eta \mu^{*}-\eta^{*} \mu},
$$

$\Delta(\mu, \nu)$ plays the role of establishing the relationship between $\eta-\xi$ phase space function and its Weyl-Wigner quantum correspondence operator. Using the similar method to deriving Eq.(21), we find that the Weyl ordering of operator $|\nu-\eta\rangle\langle\nu+\eta|$ is

$$
\begin{aligned}
|\nu-\eta\rangle\langle\nu+\eta| & ={ }^{:} \delta\left(\nu-a_{1}+a_{2}^{\dagger}\right) \delta\left(\nu^{*}-a_{1}^{\dagger}+a_{2}\right) \\
& \times \exp \left[\eta\left(\nu^{*}-2 a_{1}^{\dagger}\right)-\eta^{*}\left(\nu-2 a_{1}\right)-2\left(a_{1}^{\dagger}-\nu^{*}\right)\left(a_{1}-\nu\right)+2 a_{2}^{\dagger} a_{2}\right]:
\end{aligned}
$$

Substituting (30) into Eq.(29) and performing the integration over $d^{2} \eta$, the result is

$$
\begin{aligned}
\Delta(\mu, \nu) & ={ }^{:} \delta\left(\mu-a_{1}-a_{2}^{\dagger}\right) \delta\left(\mu^{*}-a_{1}^{\dagger}-a_{2}\right) \delta\left(\nu-a_{1}+a_{2}^{\dagger}\right) \delta\left(\nu^{*}-a_{1}^{\dagger}+a_{2}\right): \\
& \vdots \\
& \vdots \delta^{(2)}\left(\mu-a_{1}-a_{2}^{\dagger}\right) \delta^{(2)}\left(\nu-a_{1}+a_{2}^{\dagger}\right)
\end{aligned}
$$

which is also a neat Dirac's $\delta$-operator function within the Weyl ordering symbol.

As a result of Eqs.(24) and (31), we have

$$
\begin{aligned}
& \delta\left(\eta-a_{1}+a_{2}^{\dagger}\right) \delta\left(\eta^{*}-a_{1}^{\dagger}+a_{2}\right) \delta\left(\xi-a_{1}-a_{2}^{\dagger}\right) \delta\left(\xi^{*}-a_{1}^{\dagger}-a_{2}\right) \\
& =: \exp \left[\left(\xi-a_{1}-a_{2}^{\dagger}\right)\left(\eta^{*}-a_{1}^{\dagger}+a_{2}\right)-\left(\eta-a_{1}+a_{2}^{\dagger}\right)\left(\xi^{*}-a_{1}^{\dagger}-a_{2}\right)\right]: \\
& =\int \frac{d^{2} \mu d^{2} \nu}{\pi^{2}}: e^{(\xi-\mu)\left(\eta^{*}-\nu^{*}\right)-(\eta-\nu)\left(\xi^{*}-\mu^{*}\right)} \delta^{(2)}\left(\mu-a_{1}-a_{2}^{\dagger}\right) \delta^{(2)}\left(\nu-a_{1}+a_{2}^{\dagger}\right): \\
& =\int \frac{d^{2} \mu d^{2} \nu}{\pi^{2}} e^{(\xi-\mu)\left(\eta^{*}-\nu^{*}\right)-(\eta-\nu)\left(\xi^{*}-\mu^{*}\right)} \Delta(\mu, \nu) .
\end{aligned}
$$

This is the mutual transformation between the entangled form of Wigner operator $\Delta(\mu, \nu)$ and $\delta\left(\mu-a_{1}-a_{2}^{\dagger}\right)$ $\delta\left(\mu^{*}-a_{1}^{\dagger}-a_{2}\right) \delta\left(\nu-a_{1}+a_{2}^{\dagger}\right) \delta\left(\nu^{*}-a_{1}^{\dagger}+a_{2}\right)$.

Moveover, the reciprocal transformation of Eq. (32) is

$$
\begin{aligned}
& \int \frac{d^{2} \xi d^{2} \eta}{\pi^{2}} \delta\left(\eta-a_{1}+a_{2}^{\dagger}\right) \delta\left(\eta^{*}-a_{1}^{\dagger}+a_{2}\right) \delta\left(\xi-a_{1}-a_{2}^{\dagger}\right) \delta\left(\xi^{*}-a_{1}^{\dagger}-a_{2}\right) e^{-(\xi-\mu)\left(\eta^{*}-\nu^{*}\right)+(\eta-\nu)\left(\xi^{*}-\mu^{*}\right)} \\
& =\int \frac{d^{2} \xi d^{2} \eta}{\pi^{2}} \int \frac{d^{2} \mu^{\prime} d^{2} \nu^{\prime}}{\pi^{2}} \Delta\left(\mu^{\prime}, \nu^{\prime}\right) e^{\left(\xi-\mu^{\prime}\right)\left(\eta^{*}-\nu^{\prime *}\right)-\left(\eta-\nu^{\prime}\right)\left(\xi^{*}-\mu^{\prime *}\right)} e^{-(\xi-\mu)\left(\eta^{*}-\nu^{*}\right)+(\eta-\nu)\left(\xi^{*}-\mu^{*}\right)} \\
& =\int \frac{d^{2} \mu^{\prime} d^{2} \nu^{\prime}}{\pi^{2}} \Delta\left(\mu^{\prime}, \nu^{\prime}\right) e^{\left(-\nu^{\prime} \mu^{\prime *}+\mu^{\prime} \nu^{\prime *}-\mu \nu^{*}+\nu \mu^{*}\right)} \int \frac{d^{2} \xi d^{2} \eta}{\pi^{2}} e^{\xi\left(\nu^{*}-\nu^{\prime *}\right)+\xi^{*}\left(\nu^{\prime}-\nu\right)} e^{\eta^{*}\left(\mu-\mu^{\prime}\right)+\eta\left(\mu^{\prime *}-\mu^{*}\right)} \\
& =\int \frac{d^{2} \mu^{\prime} d^{2} \nu^{\prime}}{\pi^{2}} \Delta\left(\mu^{\prime}, \nu^{\prime}\right) e^{\left(-\nu^{\prime} \mu^{\prime *}+\mu^{\prime} \nu^{\prime *}-\mu \nu^{*}+\nu \mu^{*}\right)} \delta^{(2)}\left(\nu^{\prime}-\nu\right) \delta^{(2)} \delta\left(\mu^{\prime}-\mu\right) \\
& =\Delta(\mu, \nu)
\end{aligned}
$$

So Eqs. (32) and (33) are qualified to be new two-fold complex integration transformations about the Wigner operator $\Delta(\mu, \nu)$ (in its entangled form) in phase space quantum mechanics.

Further, multiplying both sides of (33) from the left by $\int \frac{d^{2} \mu d^{2} \nu}{\pi^{2}} D(\nu, \mu)$ and considering

$$
Q_{j}=\frac{a_{j}+a_{j}^{\dagger}}{\sqrt{2}}, P_{j}=\frac{a_{j}-a_{j}^{\dagger}}{i \sqrt{2}}
$$


we obtain

$$
\begin{aligned}
& \int \frac{d^{2} \mu d^{2} \nu}{\pi^{2}} D(\nu, \mu) \Delta(\mu, \nu) \\
& =\int \frac{d^{2} \xi d^{2} \eta}{\pi^{2}} \delta^{(2)}\left(\eta-a_{1}+a_{2}^{\dagger}\right) \delta^{(2)}\left(\xi-a_{1}-a_{2}^{\dagger}\right) \int \frac{d^{2} \mu d^{2} \nu}{\pi^{2}} D(\nu, \mu) e^{-(\xi-\mu)\left(\eta^{*}-\nu^{*}\right)+(\eta-\nu)\left(\xi^{*}-\mu^{*}\right)} \\
& =\int \frac{d^{2} \xi d^{2} \eta}{\pi^{2}} \delta^{(2)}\left(\eta-a_{1}+a_{2}^{\dagger}\right) \delta^{(2)}\left(\xi-a_{1}-a_{2}^{\dagger}\right) \mathcal{F}(\eta, \xi) \\
& =\int \frac{d^{2} \xi d^{2} \eta}{\pi^{2}} \delta\left(\eta_{1}-\frac{Q_{1}-Q_{2}}{\sqrt{2}}\right) \delta\left(\eta_{2}-\frac{P_{1}+P_{2}}{\sqrt{2}}\right) \delta\left(\xi_{1}-\frac{Q_{1}+Q_{2}}{\sqrt{2}}\right) \delta\left(\xi_{2}-\frac{P_{1}-P_{2}}{\sqrt{2}}\right) \mathcal{F}(\eta, \xi) \\
& =\mathcal{F}\left(\frac{Q_{1}-Q_{2}}{\sqrt{2}}, \frac{P_{1}+P_{2}}{\sqrt{2}}, \frac{Q_{1}+Q_{2}}{\sqrt{2}}, \frac{P_{1}-P_{2}}{\sqrt{2}}\right),
\end{aligned}
$$

where we have introduced

$$
\mathcal{F}(\eta, \xi) \equiv \int \frac{d^{2} \mu d^{2} \nu}{\pi^{2}} D(\nu, \mu) e^{\left(\xi^{*}-\mu^{*}\right)(\eta-\nu)-\left(\eta^{*}-\nu^{*}\right)(\xi-\mu)},
$$

which seems a new interesting transformation. Due to

$$
\begin{aligned}
& \int \frac{d^{2} \xi d^{2} \eta}{\pi^{2}} \exp \left[(\xi-\mu)\left(\eta^{*}-\nu^{*}\right)-(\eta-\nu)\left(\xi^{*}-\mu^{*}\right)\right] \\
& =\int d^{2} \xi \delta(\xi-\mu) \delta\left(\xi^{*}-\mu^{*}\right) e^{\nu\left(\xi^{*}-\mu^{*}\right)-\nu^{*}(\xi-\mu)}=1
\end{aligned}
$$

$e^{(\xi-\mu)\left(\eta^{*}-\nu^{*}\right)-(\eta-\nu)\left(\xi^{*}-\mu^{*}\right)}$ can be considered a basis function in $\xi-\eta$ phase space, or Eq. (32) can be looked as an expansion of $D(\nu, \mu)$ in terms of $e^{(\xi-\mu)\left(\eta^{*}-\nu^{*}\right)-(\eta-\nu)\left(\xi^{*}-\mu^{*}\right)}$, with the expansion coefficient being $\mathcal{F}(\eta, \xi)$.

We can prove that the inverse transform of (36) is

$$
\int \frac{d^{2} \xi d^{2} \eta}{\pi^{2}} e^{(\xi-\mu)\left(\eta^{*}-\nu^{*}\right)-(\eta-\nu)\left(\xi^{*}-\mu^{*}\right)} \mathcal{F}(\eta, \xi) \equiv D(\nu, \mu)
$$

In fact, substituting Eq. (38) into Eq. (36) yields

$$
\begin{aligned}
& \int \frac{d^{2} \xi^{\prime} d^{2} \eta^{\prime}}{\pi^{2}} \mathcal{F}\left(\eta^{\prime}, \xi^{\prime}\right) \int \frac{d^{2} \mu d^{2} \nu}{\pi^{2}} e^{\left(\xi^{\prime}-\mu\right)\left(\eta^{\prime *}-\nu^{*}\right)-\left(\eta^{\prime}-\nu\right)\left(\xi^{\prime *}-\mu^{*}\right)+\left(\xi^{*}-\mu^{*}\right)(\eta-\nu)-\left(\eta^{*}-\nu^{*}\right)(\xi-\mu)} \\
& =\int \frac{d^{2} \xi^{\prime} d^{2} \eta^{\prime}}{\pi^{2}} \mathcal{F}\left(\eta^{\prime}, \xi^{\prime}\right) e^{\left(\xi^{\prime} \eta^{\prime *}-\eta^{\prime} \xi^{\prime *}+\xi^{*} \eta-\eta^{*} \xi\right)} \int \frac{d^{2} \mu d^{2} \nu}{\pi^{2}} e^{\left(\eta^{*}-\eta^{\prime *}\right) \mu+\left(\eta^{\prime}-\eta\right) \mu^{*}} e^{\left(\xi^{\prime *}-\xi^{*}\right) \nu+\left(\xi-\xi^{\prime}\right) \nu^{*}} \\
& =\int d^{2} \xi^{\prime} d^{2} \eta^{\prime} \mathcal{F}\left(\eta^{\prime}, \xi^{\prime}\right) e^{\left(\xi^{\prime} \eta^{\prime *}-\eta^{\prime} \xi^{\prime *}+\xi^{*} \eta-\eta^{*} \xi\right)} \delta^{(2)}\left(\eta^{\prime}-\eta\right) \delta^{(2)} \delta\left(\xi-\xi^{\prime}\right) \\
& =\mathcal{F}(\eta, \xi) .
\end{aligned}
$$

Additionally, we can prove

$$
\begin{aligned}
& \int \frac{d^{2} \xi d^{2} \eta}{\pi^{2}}|\mathcal{F}(\eta, \xi)|^{2} \\
& =\int \frac{d^{2} \mu d^{2} \nu}{\pi^{2}}|D(\nu, \mu)|^{2} \int \frac{d^{2} \mu^{\prime} d^{2} \nu^{\prime}}{\pi^{2}} \exp \left[\left(\mu^{*} \nu-\nu^{*} \mu\right)+\left(\mu^{\prime} \nu^{\prime *}-\nu^{\prime} \mu^{\prime *}\right)\right] \\
& \times \int \frac{d^{2} \xi d^{2} \eta}{\pi^{2}} \exp \left[\left(\mu^{\prime *}-\mu^{*}\right) \eta+\left(\mu-\mu^{\prime}\right) \eta^{*}+\left(\nu^{*}-\nu^{\prime *}\right) \xi+\left(\nu^{\prime}-\nu\right) \xi^{*}\right] \\
& =\int \frac{d^{2} \mu d^{2} \nu}{\pi^{2}}|D(\nu, \mu)|^{2} \int d^{2} \mu^{\prime} d^{2} \nu^{\prime} \exp \left[\left(\mu^{*} \nu-\nu^{*} \mu\right)+\left(\mu^{\prime} \nu^{\prime *}-\nu^{\prime} \mu^{\prime *}\right)\right] \delta^{(2)}\left(\mu-\mu^{\prime}\right) \delta^{(2)} \delta\left(\nu^{\prime}-\nu\right) \\
& =\int \frac{d^{2} \mu d^{2} \nu}{\pi^{2}}|D(\nu, \mu)|^{2}
\end{aligned}
$$

which shows that the transformations of Eqs.(36) and (38) obey the Parseval-like theorem as well. 


\section{APPLICATION}

By using the above transformation we can solve some operator ordering problems arising from Eq.(25), for example, we want to put $\left(a_{1}^{\dagger}-a_{2}\right)^{n}\left(a_{1}+a_{2}^{\dagger}\right)^{m}$ into its Weyl ordered form. According to Eqs.(8), (13) and (15), we have

$$
\frac{1}{2} \int \frac{d^{2} \eta d^{2} \xi}{\pi^{2}}|\eta\rangle\langle\xi| e^{\left(\eta^{*} \xi-\eta \xi^{*}\right) / 2}=1
$$

and

$$
\frac{1}{2} \int \frac{d^{2} \eta d^{2} \xi}{\pi^{2}}|\xi\rangle\langle\eta| e^{\left(\eta \xi^{*}-\eta^{*} \xi\right) / 2}=1
$$

As a result of Eqs.(41) and (23), using the eigenvector equations Eqs.(6) and (11), we obtain

$$
\begin{aligned}
& \left(a_{1}^{\dagger}-a_{2}\right)^{n}\left(a_{1}+a_{2}^{\dagger}\right)^{m} \\
& =\frac{1}{2} \int \frac{d^{2} \eta d^{2} \xi}{\pi^{2}} \eta^{* n} \xi^{m}|\eta\rangle\langle\xi| e^{\left(\eta^{*} \xi-\eta \xi^{*}\right) / 2} \\
& =\int \frac{d^{2} \eta d^{2} \xi}{\pi^{2}} \eta^{* n} \xi^{m}: \exp \left[\left(\xi-a_{1}-a_{2}^{\dagger}\right)\left(\eta^{*}-a_{1}^{\dagger}+a_{2}\right)-\left(\eta-a_{1}+a_{2}^{\dagger}\right)\left(\xi^{*}-a_{1}^{\dagger}-a_{2}\right)\right]_{:}^{:} \\
& =\int \frac{d^{2} \eta d^{2} \xi}{\pi^{2}} \sum_{k=0}^{n} \sum_{l=0}^{m} \frac{i^{n+m} m ! n !}{(n-k) ! k !(m-l) ! l !} \eta_{1}^{n-k}\left(-\eta_{2}\right)^{n} \xi_{1}^{m-l} \xi_{2}^{m} \\
& \times: \exp \left\{\left[-2 i \eta_{2}+\left(a_{1}+a_{2}-a_{2}^{\dagger}-a_{1}^{\dagger}\right)\right]\left[\xi_{1}-\frac{1}{2}\left(a_{2}^{\dagger}+a_{1}^{\dagger}+a_{1}+a_{2}\right)\right]\right. \\
& \left.+\left[2 i \eta_{1}+i\left(a_{2}^{\dagger}-a_{1}^{\dagger}-a_{1}+a_{2}\right)\right]\left[\xi_{2}-\frac{1}{2} i\left(a_{1}^{\dagger}-a_{1}+a_{2}-a_{2}^{\dagger}\right)\right]\right\}: \\
& =-\sum_{k=0}^{n} \sum_{l=0}^{m} \frac{i^{n+m} m ! n !}{(n-k) ! k !(m-l) ! l !} \\
& \times \int \frac{d\left(-\eta_{2}\right) d \xi_{1}}{\pi}\left(-\eta_{2}\right)^{n} \xi_{1}^{m-l}: \exp \left\{2 i\left[-\eta_{2}+\frac{1}{\sqrt{2}}\left(P_{1}+P_{2}\right)\right]\left[\xi_{1}-\frac{1}{\sqrt{2}}\left(Q_{1}+Q_{2}\right)\right]\right\} \\
& \times \int \frac{d \eta_{1} d \xi_{2}}{\pi} \eta_{1}^{n-k} \xi_{2}^{m} \exp \left\{2 i\left[\eta_{1}-\frac{1}{\sqrt{2}}\left(Q_{1}-Q_{2}\right)\right]\left[\xi_{2}-\frac{1}{\sqrt{2}}\left(P_{1}-P_{2}\right)\right]\right\}: \\
& =-\left(\frac{1}{2}\right)^{n+m} \sum_{k=0}^{n} \sum_{l=0}^{m} \frac{m ! n !(\sqrt{2})^{k+l}}{(n-k) ! k !(m-l) ! l !} \\
& \times{ }_{:}^{:} H_{n-k, m}\left[\left(Q_{1}-Q_{2}\right), i\left(P_{1}-P_{2}\right)\right] H_{m-l, n}\left[i\left(Q_{1}+Q_{2}\right),-\left(P_{1}+P_{2}\right)\right]:
\end{aligned}
$$

where we have used the following integration formula 12$]$

$$
\int \frac{d x d y}{\pi} x^{m} y^{r} \exp [2 i(y-s)(x-t)]=\left(\frac{1}{\sqrt{2}}\right)^{m+r}(-i)^{r} H_{m, r}(\sqrt{2} t, i \sqrt{2} s),
$$

with $H_{m, r}$ is the two-variable Hermite polynomials [13],

$$
H_{m, r}(t, s)=\sum_{l=0}^{\min (m, r)} \frac{m ! r !(-1)^{l}}{l !(m-l) !(r-l) !} t^{m-l} s^{r-l} .
$$


Eq.(43) is a simple approach to turn $\left(a_{1}^{\dagger}-a_{2}\right)^{n}\left(a_{1}+a_{2}^{\dagger}\right)^{m}$ into its Weyl ordering. Similarly, using Eqs.(27) and (42), we see that the Weyl ordered form of $\left(a_{1}+a_{2}^{\dagger}\right)^{m}\left(a_{1}^{\dagger}-a_{2}\right)^{n}$ is

$$
\begin{aligned}
& \left(a_{1}+a_{2}^{\dagger}\right)^{m}\left(a_{1}^{\dagger}-a_{2}\right)^{n} \\
& =-\left(\frac{1}{2}\right)^{m+n} \sum_{k=0}^{n} \sum_{l=0}^{m} \frac{(-1)^{l} m ! n !(\sqrt{2})^{k+l}}{(n-k) ! k !(m-l) ! l !} \\
& \times: H_{n, m}\left[\left(Q_{2}-Q_{1}\right), i\left(P_{1}-P_{2}\right)\right] H_{m-k, n-l}\left[\left(Q_{1}+Q_{2}\right), i\left(P_{1}+P_{2}\right)\right]
\end{aligned}
$$

In summary, as a natural extension of our preceding paper [7], through employing operators' Weyl ordering expansion the formula we have found a new two-fold complex integration transformation about the Wigner operator $\Delta(\mu, \nu)$ (in its entangled form) in phase space quantum mechanics, which is invertible and obeys Parseval theorem. The new complex integration transformation is compatible to $\eta-\xi$ phase space quantum mechanics which takes entangled state representations as basis. Under this transformation the relationship between the Weyl ordering and $\left(a_{1}^{\dagger}-a_{2}\right) \Leftrightarrow$ $\left(a_{1}+a_{2}^{\dagger}\right)$ ordering or $\left(a_{1}+a_{2}^{\dagger}\right) \Leftrightarrow\left(a_{1}^{\dagger}-a_{2}\right)$ ordering of operators is revealed. In this way, the contents of phase space quantum mechanics[5] can be further enriched. Studies about the multi-mode case are in progress and new results will be reported later.

[1] H. Y. Fan and L. Y. Hu, "Optical transformation from chirplet to fractional Fourier transformation kernel," J. Mod. Opt. 56, 1227-1229 (2009).

[2] H. Y. Fan, "Weyl ordering quantum mechanical operators by virtue of the IWWP technique," J. Phys. A Math. Gen. 25, 3443-3447 (1992).

[3] H. Y. Fan, "Newton-Leibniz integration for ket-bra operators in quantum mechanics (IV)-Integrations within Weyl oedered product of operators and their applications," Ann. Phys. 323, 500-526 (2008).

[4] E. Wigner, "On the Quantum Correction For Thermodynamic Equilibrium" Phys. Rev. 40, 749-759 (1932).

[5] W. P. Schleich, Quantum Optics in Phase Space, Wiley-VCH, Berlin and many references therein (2001).

[6] H. Y. Fan, "Operator ordering in quantum optics theory and the development of Dirac's symbolic method" J. Opt. B: Quantum Semiclass. Opt. 5, R147-R163 (2003).

[7] H. Y. Fan, "Wigner operator's new transformation in phase space quantum mechanics and its applications" arXiv: 0903.1769vl [quanut-ph] (2009).

[8] A. Einstein, B. Podolsky and N. Rosen "Can quantum mechanical description of physical reality be considered complete?" Phys. Rev. 47, 777-780 (1935).

[9] H. Y. Fan and J. R. Klauder "Eigenvectors of two particles' relative position and total momentum," Phys. Rev. A 49, 704-707 (1994).

[10] R. J. Glauber, "The Quantum Theory of Optical Coherence" Phys. Rev. 130, 2529-2539 (1963); "Coherent and Incoherent States of the Radiation Field" Phys. Rev. 131, 2766-2788 (1963).

[11] H. Y. Fan, "Time evolution of the Wigner function in the entangled-state representation," Phys. Rev. A 65, 064102 (2002).

[12] H. Y. Fan, "A new kind of two-fold integration transformation in phase space and its uses in Weyl ordering of operators" Comm. Theor. Phys. 50, 935-937 (2008).

[13] A. Erdèlyi, Higher Transcendental Functions (The Bateman Manuscript Project, McGraw Hill 1953). 